Recepción: 31/ 10 / 2016

Aceptación: 28 / 02 / 2017

Publicación: 29 / 04 / 2017

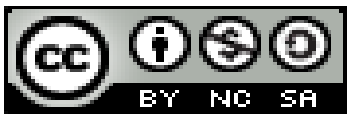

Ciencias de la salud

Artículo de investigación

\title{
Algunas consideraciones actuales acerca de la calidad de los servicios odontológicos
}

Some current considerations about the quality of dental services

\section{Algumas considerações atuais sobre a qualidade de serviços odontológicos}

\author{
Alba M. Mendoza-Castro ${ }^{\mathrm{I}}$ \\ albamariamc@hotmail.com \\ Julio C. Jimbo-Mendoza ${ }^{\mathrm{II}}$ \\ juliojimbomendoza@gmail.com \\ Freddy A. Ávila-Chica ${ }^{\mathrm{III}}$ \\ freddyavilach@gmail.com
}

Correspondencia: albamariamc@hotmail.com

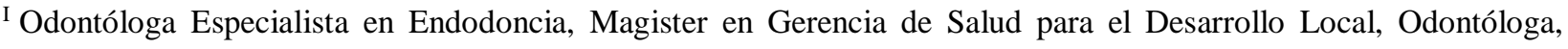
Docente de la Universidad Laica Eloy Alfaro de Manabí, Manta, Ecuador.

II Magister en Gerencia de Salud para el Desarrollo Local, Especialista en Gerencia y Planificación Estratégica en Salud, Doctor en Odontología, Diplomado Superior en Odontología Integral, Diploma Superior en Desarrollo Local y Salud, Docente de la Universidad Laica Eloy Alfaro de Manabí, Manta, Ecuador.

III Magister en Salud Publica Mención Gerencia de los Servicios de Salud, Doctor en Odontología, Diplomado Superior en Educación Universitaria por Competencias, Docente de la Universidad Laica Eloy Alfaro de Manabí, Manta, Ecuador.
} 


\title{
Resumen
}

En lo relacionado con los servicios, calidad no significa ni lujo, ni la condición superior en la prestación asistencial, ni suministrar la apariencia externa, ni tampoco proporcionar la máxima comodidad; hoy es considerada como el nivel de excelencia que la empresa ha escogido y se ha impuesto como norma, para satisfacer las necesidades del cliente. La siguiente revisión bibliográfica se basa sobre los nuevos criterios de la calidad de los servicios odontológicos, donde se exponen algunos conceptos de calidad y el método de evolución de la misma, para poder entonces mejorar la atención de los pacientes y su más completa satisfacción.

Palabras clave: calidad de la atención, evaluación, servicios odontológicos.

\begin{abstract}
In terms of services, quality does not mean luxury, nor the superior condition in the provision, nor provide the external appearance, nor give the maximum comfort. Today is considered frankly and plainly, the level of excellence that the company has chosen and imposed as a standard, to meet the needs of the customer. The following literature review is based on the new criteria for the quality of dental services. This document presents some concepts of quality, method of evolution of the same to be able to improve patient care and satisfaction.
\end{abstract}

Key words: quality of care, evaluation, dental services.

\section{Resumo}

Em relação aos serviços, a qualidade não significa nem luxo nem condição superior na prestação de cuidados, ou fornecer aparência externa, nem proporcionar o máximo conforto; hoje é considerado o nível de excelência que a empresa escolheu e foi imposta como regra, para atender às necessidades dos clientes. A seguinte revisão da literatura baseia-se nos novos critérios de qualidade de serviços odontológicos, onde alguns conceitos de qualidade e método de evolução do mesmo, para, em seguida, melhorar o atendimento ao paciente e satisfação completa estão expostos. 
Palavras chave: calidad de la atención, evaluación, servicios odontológicos.

\section{Introducción}

Existe diversidad de conceptos en relación con la calidad de la atención en salud. Diferentes autores, como Carnota Lauzan, Otero, Marracino, entre otros, aportaron criterios al respecto, pero el más aceptado es el de Avedis Donabedian, la máxima autoridad en el campo de la calidad de atención médica, quien la define como los logros de los mayores beneficios posibles de la atención médica con los menores riesgos para los pacientes. También se tiene muy en cuenta la definición de la Organización Mundial de la Salud (OMS), que la define como un alto nivel de excelencia profesional, relacionada con el uso eficiente de los recursos, con el mínimo de riesgos para el paciente y un alto grado de satisfacción por parte de este (impacto final en la salud). ${ }^{1,2}$

Desde los primeros estados de la esclavitud comenzó el estudio de la calidad, por lo cual fue motivo de preocupación social. Se destacaron por sus aportes, Kauro Ishikawa, Joseph M. Juran y Edgard Deming, provenientes del sector empresarial; los dos últimos introdujeron los principios de administración de la calidad en la industria japonesa. Por su parte, Pareto y otros, son considerados los garúes de la calidad empresarial. ${ }^{3}$

Se enfatiza en la calidad como categoría indispensable para lograr la satisfacción de quienes brindan o reciben un determinado servicio, así como en los aspectos que deben tenerse en cuenta para garantizar el éxito de la gestión. Para la OMS, la calidad permite asegurar que cada paciente reciba el conjunto de servicios diagnósticos y terapéuticos más adecuados para conseguir una atención sanitaria óptima, con mínimo riesgo y máxima satisfacción. ${ }^{4}$

\section{Calidad en los servicios odontológicos}

Las investigaciones en calidad del servicio en hospitales y en clínicas dentales, están estrechamente relacionadas por las similitudes de los servicios. La calidad de la atención odontológica brindada en las clínicas dentales se ha convertido en una necesidad para todo profesional de la salud, ya sea práctica privada o salubrista. ${ }^{5}$ 
Son poco los estudios que se refieren concretamente a la atención odontológica, y menos aun a su evaluación, asumiendo que al hablar de la atención médica, la odontología queda implícita. No obstante es necesario el abordaje de la calidad en la odontología, por cuanto esta disciplina, considerada por algunos como una especialidad dentro de la medicina, tiene sus propias características y sus particularidades. ${ }^{5}$

En ese mismo orden y dirección, es posible que el odontólogo considere que no tiene problema y que el paciente quedó satisfecho, aparentemente, con la atención recibida, y se ha podido confirmar por ello de que ha existido realmente calidad en la atención, pero en algunos casos consideramos que no. En observaciones realizadas existieron algunas fallas en el proceso de atención sin identificar las causas, puesto que no son el resultado de inadecuados diagnósticos o tratamiento.

Existen preguntas como "¿el paciente espera mucho tiempo para ser atendido”, “¿el paciente no ha modificado su comportamiento referente a la higiene bucal? Las respuestas a estas preguntas y a otras indicarán si existen dificultades con la planificación del trabajo, con la organización, con el clima organizacional y la comunicación profesional/paciente. ${ }^{5}$

Las dimensiones de la calidad desempeñan un papel importante en la evaluación de dicho proceso; sin embargo, uno de los problemas principales para la interpretación más acertada de lo que sucede es lograr no solo el análisis de cada una de ellas en un escenario dado, sino entender su interrelación y en qué medida, por ejemplo, un buen resultado se asocia a procesos correctos, o una excelente estructura ha desencadenado o no procesos y resultados de alta calidad. Hasta ahora parece, que no existe certeza que la excelencia en una dimensión implique lo mismo en las restantes. $^{4}$

Para evaluar la calidad se han utilizado varias herramientas, como monitoreos, modelos, instrumentos y propuestas metodológicas, como la de Jiménez Cangas, de la Escuela Nacional de Salud Pública de Cuba, la cual se confeccionó en un inicio para la atención primaria, y hoy es utilizada para todos los niveles. ${ }^{6}$

El enfoque en sistema, planteado desde hace algunos años por Donabedian, es aceptado de forma generalizada. De acuerdo con ese enfoque, la calidad se estudia y se mide teniendo en cuenta tres componentes esenciales: estructura, proceso y resultados. ${ }^{5}$ 
Estructura: Este componente se refiere a la cantidad y calificación del personal, los equipos e instrumentos disponibles, los recursos financieros, las instalaciones y otros recursos materiales, las normas y otros reglamentos existentes; así como la organización establecida. A nivel más general incluye la voluntad política y el liderazgo comprometido, como también la formulación de políticas y estrategias. En otras palabras, se refiere al conjunto de atributos de índole organizativo o material, que se mantiene con relativa estabilidad temporal. ${ }^{5}$

Proceso: Se refiere al conjunto de actividades que se realizan en la atención, y aquellas que se desarrollan para asegurar la ejecución del propio proceso. Dentro de este componente se incluyen no solo las acciones ejecutadas por el personal de salud, con el nivel de destreza y habilidad de que dispongan, sino también las que puedan desplegar los propios pacientes. ${ }^{5}$

Resultados: En última instancia es la consecuencia que tiene el proceso de atención en el subsiguiente cambio en el estado de salud. Por otra parte incluye el análisis del nivel de impacto; el cumplimiento de indicadores y de los gastos efectuados, la satisfacción de los usuarios, prestadores y decisores, la certificación y acreditación institucional, así como la identificación de nuevos problemas que llevan a un perfeccionamiento continuo. ${ }^{5}$

Los componentes en que los distintos autores fundamentan la medición de la calidad para su mejoramiento, se pueden analizar a partir de las definiciones que se han dado sobre la de calidad de la atención, por los métodos que recomiendan y por los datos que emplean. ${ }^{5}$

Actualmente se utilizan más los métodos que toman datos de proceso y resultados, o de los tres componentes, toda vez que se ha visto claramente que una buena estructura no asegura un buen proceso, ni que un buen proceso asegura un buen resultado, ni tampoco que un buen resultado puede asegurar las bondades de la estructura o del proceso. De ahí la importancia que se da hoy al enfoque sistémico y la tendencia a no considerar aisladamente un componente. ${ }^{5}$

Por las consideraciones anteriores, en la atención en odontología, cada vez se tiende más a la medición de los resultados como aspectos cruciales de la evaluación de los servicios. Todas las acciones deben ser objeto de medición, para que se puedan evaluar cuantitativamente los procesos, lo que permite construir deducciones e interrogantes, comparaciones en tiempo y espacio, mucho más precisas y menos dependientes de la subjetividad del observador. ${ }^{7}$ 
Gonzalo H. en 2004 estudió el nivel de satisfacción de los pacientes atendido en la clínica odontológica de la UNMMSM, encontrando un nivel de aceptación de los servicios catalogado como bueno y muy bueno en cuanto a infraestructura y equipamiento. Un gran número de pacientes $(33,7 \%)$ manifestó insatisfacción con el tiempo de espera, pero estuvieron satisfechos con el trato recibido. ${ }^{8,9}$

Jacinto Núñez ${ }^{9}$ en su investigación realizada en la Clínica de la Facultad de Odontología UMNSM, Lima Perú, con vistas a determinar la percepción de la calidad de la atención, los resultados en las dimensiones estructura y proceso arrojaron que los usuarios perciben el servicio como malo, y en la dimensión resultado lo califican de bueno, mayoritariamente, lo cual pone de manifiesto los componentes subjetivo y objetivo de la calidad. Los pacientes se sienten bien tratados (componente subjetivo); sin embargo, desconocen una serie de acciones, habilidades y conocimientos que deben desarrollar los prestadores en su desempeño profesional para poder demostrar que han brindado un servicio de calidad (componente objetivo).

Otro estudio realizado por Coria Fierros et al. con respecto a la calidad de la atención odontológica en las clínicas Iztacala, Acatlán y Aragón dependientes de la Fes Iztacala, arrojó resultados positivos, estableciendo un grado alto de satisfacción de los pacientes que intervinieron en este estudio, que calificaron a los servicios odontológicos humanos

En sentido general, para tener la calidad de los servicios odontológicos, ya sea en un consultorio dental en una clínica que trabajen varios profesionales o algún otro servicio, es necesario tener en cuenta todos los elementos expuestos.

\section{Referencias bibliográficas}

1- Carnota Lauzan, O. “Garantía de calidad en salud”. En: Tecnologías gerenciales. Una oportunidad para los servicios de salud. La Habana: Editorial Ciencias Médicas; 2005, p. 171209.

2- Torres, M. Modelos de evaluación de la calidad del servicio. INFOcalSER: investigación en calidad del servicio, información y productividad, 2008 [citado 13 sep. 2016]. Disponible en: http://infocalser.blogspot.com/2008/07/modelos-de-evaluacin-de-la-calidad-del.htm 
3- Sánchez Jacas, I. "El diseño metodológico de las investigaciones evaluativas relacionadas con la calidad en salud. 2016". Revista Medisan [citado 13 sep. 2016]; 20(4). Disponible en: http://www.medisan.sld.cu/index.php/san/article/view/812/html

4 Revilla Arias, Haydée; González Mustelier, Denny; Valenzuela Fonseca, Leydys Magda. "Calidad de la atención de salud brindada en un servicio de rehabilitación integral de base comunitaria". MEDISAN 2016 [citado 13 sep. 2016] ; 20( 3 ): 313-319. Disponible en: http://scielo.sld.cu/scielo.php?script=sci_arttext\&pid=S1029-30192016000300006\&lng=es.

5- Rosales Sosas, M. “Cómo lograr la calidad en la calidad dental”. [citado 19 Mayo 2017]. Disponible en: www.sld.cu/galerias/pdf/uvs/saludbucal/artcalidad.pdf

6- Jiménez Cangas, L.; Báez Dueñas, R. M.; Pérez Maza, B.; Reyes Álvarez, I. "Metodología para la evaluación de la calidad en instituciones de atención primaria de salud". Revista Cubana Salud Pública. 1996 [citado 13 sep. 2016]; 22(1):15-6. Disponible en: http://scielo.sld.cu/scielo.php?script=sci_arttext\&pid=S0864-34661996000100008\&lng=es

7- Valdivieso, A. Calidad En Servicios Odontológicos. 2010. [citado 13 sep. 2016]. Disponible en: https://es.slideshare.net/anickjackie/calidad-en-servicios-odontologicos

8- Gonzalo, H. "Nivel de satisfacción de los pacientes atendidos en la Clínica Odontológica de la UNMSM 2004”. 2005. Universidad Normal Mayor de San Marcos

9- Jacinto Núñez, J. "Percepción de la calidad de atención del paciente que acude a la Clínica de la Facultad de Odontología de la UNMSM durante el año 2007” 2008. [citado 13 sep. 2016]. Disponible en: cybertesis.unmsm.edu.pe/bitstream/cybertesis/2228/1/Jacinto_nj.pdf

10- Coria Fierros, X.; Guzmán Sandoval, V. S.; Hernández Hernández, E.; Mares Valencia, S.; Martínez Alcántara, R.; Sosa Fernández, M. "La calidad en la atención odontológica de las clínicas Iztacala, Acatlán y Aragón dependientes de la Fes Iztacala”. 2003. [citado 13 sep. 2016].

Disponible

en: http://odontologia.iztacala.unam.mx/memorias $15 \mathrm{col} /$ contenido/cartel/Calidadenlaatencartel60 .htm 